\title{
Economic viability outcome of assisted reproduction technology setup: Mathematical model
}

\author{
Hamza A. Eskandarani ${ }^{1,2}$ \\ ${ }^{1}$ Department of Medical Biochemistry, College of Medicine, University of Dammam, Dammam, Saudi Arabia \\ ${ }^{2}$ Banoon Assisted Reproduction Technology Centre, The Military Hospital, Bahrain Defence Force, Riffa, Bahrain \\ Email: heskandarani@ud.edu.sa
}

Received 2 October 2012; revised 3 November 2012; accepted 12 November 2012

\begin{abstract}
Objective: To develop an evaluation method measuring the economic viability outcome of assisted reproductive technology (ART) setup in a concerted fashion. Methods: A mathematical model namely; economic viability outcome (EVO) value has been purposely constructed. The model consists of three important domains: 1) Economic performance outcome of the setup; 2) Sustainability outcome; and 3) Control outcome. The model was put to the test at Banoon ART centre, the military hospital in Bahrain during the period Sep 2004 to Sep 2006. Results: When this model was stringently applied at the said centre, the EVO value was increased by $40 \%$ from 0.84 to 1.40 within 12 months of rectifications which started Sep 2005. Even though the economic performance has dropped by approximately $22 \%$, due to an extra spending towards assets and lower utilization of treatment cycles, the centre showed improvements at various levels as evident from the resulting higher EVO value. Conclusions: EVO value as a model provides a mechanism by which ART setup can be economically evaluated and self-assessed. It can also generate a method for measuring the safety and efficacy of the treating centre.
\end{abstract}

Keywords: Economic Viability Outcome Value; ART Setup; Economic Performance; Success Rate Variance; Compliance Factor

\section{INTRODUCTION}

Assisted reproductive technology has been used as the effective mean of treating infertility which affects $10 \%$ $15 \%$ of couples worldwide [1,2]. ART employs various technological procedures amongst which are in vitro fertilization (IVF) and intracytoplasmic sperm injection (ICSI) cycles, intrauterine insemination (IUI), testicular extraction (TESE), and cryopreservation of gametes and embryos. However, ART procedures have still not found their way into the mainstream of state funded health care and exist mainly in the private sector [3]. Treatment cycles, which involve IVF/ICSI procedures, are usually costly (Table 1). As a result, these forms of treatment may be beyond the financial means of many deserving couples who may subject themselves to less effective but available therapy such as tubal surgery for severely damaged fallopian tubes. Where there is some National Health Service provision for assisted conception, funding is severely limited which can lead to rationing of treatment based on factors such as age and the number of previous children [4].

Many IVF centres have been established round the world and as the need for infertility treatment services increased, the demand of opening new centres has picked up from its long hiatus at a frantic pace. However, this booming technological setup was not matched by enough performance outcomes studies and their relevant costeffectiveness role which highlight the importance of the vitality of ART setup; thus reflecting on how well the concerned centres were performing. Such important studies warrant the need for a measurable entity which depends on parameters for evaluation and self-assessment.

\section{Planning of ART Investment}

The need of ART investment in a particular region is dependent on the percentage of infertility cases amongst couples as well as the population in the region. While, the driving force for the success of the investment is proportional to the success rates of both pregnancy and live birth at that particular ART setup. Nevertheless, the more centres that are present in a particular region the better the chance available for patients to receive treatments. However, this may have its drawbacks as competition between centres for limited patients may drive some centres to compromise on quality of offered services that often require continuous technological advancement. Such circumstances dictate the appropriate method for evaluating performance of various outcomes 
Table 1. Relative treatment cycles and their costs in different countries.

\begin{tabular}{|c|c|c|c|c|c|c|c|c|}
\hline Country & $\begin{array}{l}\text { Population } \\
\text { (millions) }\end{array}$ & GDP/capita \$ & $\mathrm{IM} / 1000$ & $\begin{array}{l}\text { \#ART } \\
\text { centres }\end{array}$ & $\begin{array}{l}\text { IVF/ICSI } \\
\text { cycles }^{* *}\end{array}$ & $\begin{array}{c}\text { IVF/ICSI } \\
\text { cycles/centre }\end{array}$ & $\begin{array}{c}\text { IVF/ICSI } \\
\text { cycles/million }\end{array}$ & $\begin{array}{c}{ }^{*} \text { Cost }(\$) \text { per } \\
\text { cycle }\end{array}$ \\
\hline USA & 302 & 41,400 & 6.5 & 490 & 170,000 & 347 & 563 & 12,000 \\
\hline UK & 61 & 30,400 & 5.2 & 85 & 35,000 & 412 & 574 & 7000 \\
\hline Japan & 127 & 30,600 & 2.8 & 650 & 100,000 & 154 & 787 & 8000 \\
\hline Bahrain & 0.7 & 21,600 & 17.3 & 5 & 700 & 140 & 1000 & 2000 \\
\hline Qatar & 0.8 & 31,400 & 18.6 & 2 & 1100 & 550 & 1375 & 2500 \\
\hline Kuwait & 2.7 & 16,300 & 10 & 8 & 1400 & 175 & 519 & 3500 \\
\hline UAE & 4.4 & 27,960 & 14.5 & 9 & 2500 & 278 & 568 & 4000 \\
\hline Saudi & 25 & 15,200 & 13.2 & 28 & 7500 & 268 & 300 & 3000 \\
\hline Jordan & 6 & 5100 & 17.4 & 18 & 7000 & 389 & 1167 & 1700 \\
\hline
\end{tabular}

*Average cost per IVF/ICSI cycle without medications, consultations, and screening. ${ }^{* *}$ Data collected from personal communications with market research departments of Medicult, Ferring and Organon companies.

as presented in this study.

\section{METHODS}

\subsection{Construction of the Mathematical Model}

In any medical service organization outcomes play a pivotal role in showing the level of care and patient's satisfaction, and the extent of the economic viability of enterprise. Nevertheless, economic performance and viability outcomes should be integrated into all aspects of the organization. Hence, economic viability outcome can be projected as a model on how to manage an organization for results and to continuously improve results. In turn, better results mean that more infertile couples are being helped in the society. We have, therefore, proposed "economic viability outcome (EVO) value" for ART setup which is proportional to the outcomes of economic performance $(\mathrm{P})$ and the success rate $(\mathrm{S})$ of the setup.

\section{i.e. $\mathrm{EVO} \propto \mathrm{PS}$.}

Hence EVO value equals the economic performance outcome of the ART setup multiplied by the outcomes of the driving force or sustainability (proportion of success) and level of compliance to standards (control).

EVO

$=$ Economic Performance $\times$ Sustaninability $\times$ Control

EVO value can be determined in the following equation when applying certain assumptions as explained in the two consecutive paragraphs (assumptions and key values of the model) shown below:

$$
\begin{aligned}
\text { EVO }= & \frac{\text { Annual Revenue }}{\text { Expenses }} \times \text { Proprotion of Success } \\
& \times \text { Compliance Factor }
\end{aligned}
$$

$$
\begin{aligned}
\text { EVO } & =\frac{\text { No of Cycles } \times \text { Cost of cycle }}{\text { Asset }+ \text { Annual Expenditure }} \\
& \times \text { Clinical Pregnancy }
\end{aligned}
$$

\subsection{Assumptions Used in the Model}

The postulated economic viability outcome value is indicative of setup viability to thrive in a given region and at a particular timeframe. Therefore, any change in conditions may result in a concomitant change in value. Nevertheless, EVO value is determined from the following three main domains:

1) Economic performance which is the ratio of annual revenue generated from the investment over asset and annual expenditure. Revenue is mainly generated from annually performed completed IVF/ICSI cycles which include surgical procedures, laboratory investtigations, consultations, and medications (other ART procedures such as IUI and TESE are considered subsidiary procedures and can be taken into consideration subject to frequency of utilization). On the other hand, asset is the fixed cost paid to secure all necessary and required functionality of the centre excluding expenditure which is the running cost paid for salaries, consumables, operation and maintenance, security, insurance, advertisement, and other needed supplies. However, the cost of investment is expected to be lower if the setup is part of a hospital as utilizetion of facilities cuts down extra cost. Nevertheless, good return of the investment allows better development and expansion of business to probably include new programs such as PGD and/or research study.

2) Proportion of success which is calculated as the number of clinical pregnancies to the power of live birth rate in an ART setup. This could certainly be used as 
self-assessment to the progress of the work. Since improvement in pregnancy rate of the centre will raise the average pregnancy rate of the relevant region. Hence, excellent live birth rate of the centre should increase the resulting EVO value. The determining factor in the success proportion is not how many clinical pregnancies obtained by the setup but by the live birth rate, which is crucial.

3) The compliance factor which is a measure of adherence to set standards by the statutory body in a given region. It has an estimated value between $0-1$. This can be calculated from a checklist to the required standard set by local authority, international standards, and/or improvised own standards. Value $=1$ means that the ART setup is fully complaint to imposed standards, while very low value could be associated with legal issues which may pose ethical problems. Table 2 devises checklists of previously presented sets of ART regulations and guidelines pertained to a specific region $[5,6]$.

However, the proposed mathematical model was put to the test at Banoon ART centre, the military hospital in Bahrain during the period Sep 2004 to Sep 2006.

\subsection{Statistical Analysis}

Data was analyzed using SPSS software package version 12.0. The level of significance was set at $\mathrm{P}<0.05$. The paired t-test was used for comparison of scores for the legalization to practice and for treatment cycles of the centre before and after the rectifications.

\section{RESULTS}

\subsection{Testing the Model}

The model is assumed to work on any ART setup so long as the parameters are factual in the given region and any comparative calculations should be carried out within the study group of setups within the localized region. This thus satisfies the same conditions. However, if conditions were different as the settings would also be different, then comparative calculations of EVO value would be biased. The model has been subjected to the test in a small region, like Bahrain, where there were three licensed ART centres in addition to two new centres that were commissioned to operate in June 2006. The integrated economic performance outcomes values were, therefore, calculated as shown below. The generated values were based on outcomes of complete (including consultations, ultrasounds, medications, and laboratory tests) IVF/ICSI cycles only which constituted more than $90 \%$ of all offered services.

\subsection{Original Setting}

The ART centre under test has been providing infertility treatments based on various aspects of assisted reproductive technologies. From Sep 2004 till Sep 2005, the centre performed 195 completed IVF/ICSI cycles (for women ages 25 - 40 years) which resulted in 40 clinical pregnancies and ended in 17 deliveries (Table 3) [7]. The average annual pregnancy rate and live birth rate was, therefore, $20.5 \%$ and $8.7 \%$ respectively. However, there was no published annual national pregnancy rate for Bahrain as the other ART centres were not releasing their ART registry data. During that year the centre spent $\$ 50,000$ as extra asset cost. The annual depreciation value was kept at $\$ 50,000$ as the expected life-time durability of most equipment was 5 years. However, the annual expenditure was approximately $\$ 650,000$. The approximate average cost of IVF/ICSI cycle including medications and tests was $\$ 4500$. The centre has scored 0.52 of the compliance factor $\left(\mathrm{C}_{\mathrm{f}}\right)$ value from a check list of devised standards (Table 2).

$$
\begin{aligned}
& \mathrm{EVO}_{1} \\
& =\frac{195 \times(\$) 4500}{(\$) 50,000+50,000+650,000} \times 40^{(8.7 / 100)} \times 0.52 \\
& \mathrm{EVO}_{1}=1.17 \times 1.38 \times 0.52 \\
& \mathrm{EVO}_{1}=0.84
\end{aligned}
$$

\subsection{Revamped Setting}

Following rigorous improvements at various levels of the setup — included acquiring new equipment, installation of uninterrupted power supply system, renovations to suite international standards, revised policies and procedures, employing more experienced and trained staff, and utilizeing updated techniques - the centre showed a remarkable change in tangible results and patients satisfaction within few months of initiation of the amendment work which started Sep 2005 and lasted for few months. Such improvements incurred an extra asset cost of $\$ 150,000$ while maintaining the same running costs at $\$ 650,000$ annually without imposing surcharge on treatment cycles, but discounted by approximately 11\%. From Sep 2005 till Sep 2006, the centre carried out 182 completed IVF/ ICSI cycles (for women ages 25 - 40 years) which resulted in 44 clinical pregnancies and ended in 27 deliveries (Table 4) [7]. The average annual pregnancy rate and live birth rate increased to $24.2 \%$ and $14.8 \%$ respectively. The centre scored $\mathrm{C}_{\mathrm{f}}$ value of 0.88 . The expected drop in the number of cycles was due to the opening of two additional ART centres in the region. Therefore, $\mathrm{EVO}_{2}$ value is calculated as:

$$
\begin{aligned}
& \mathrm{EVO}_{2} \\
& =\frac{182 \times(\$) 4000}{(\$) 150,000+50,000+650,000} \times 44^{(14.8 / 100)} \times 0.88
\end{aligned}
$$


Table 2. Checklist related to the legalization to practice and treatment cycles of ART centre.

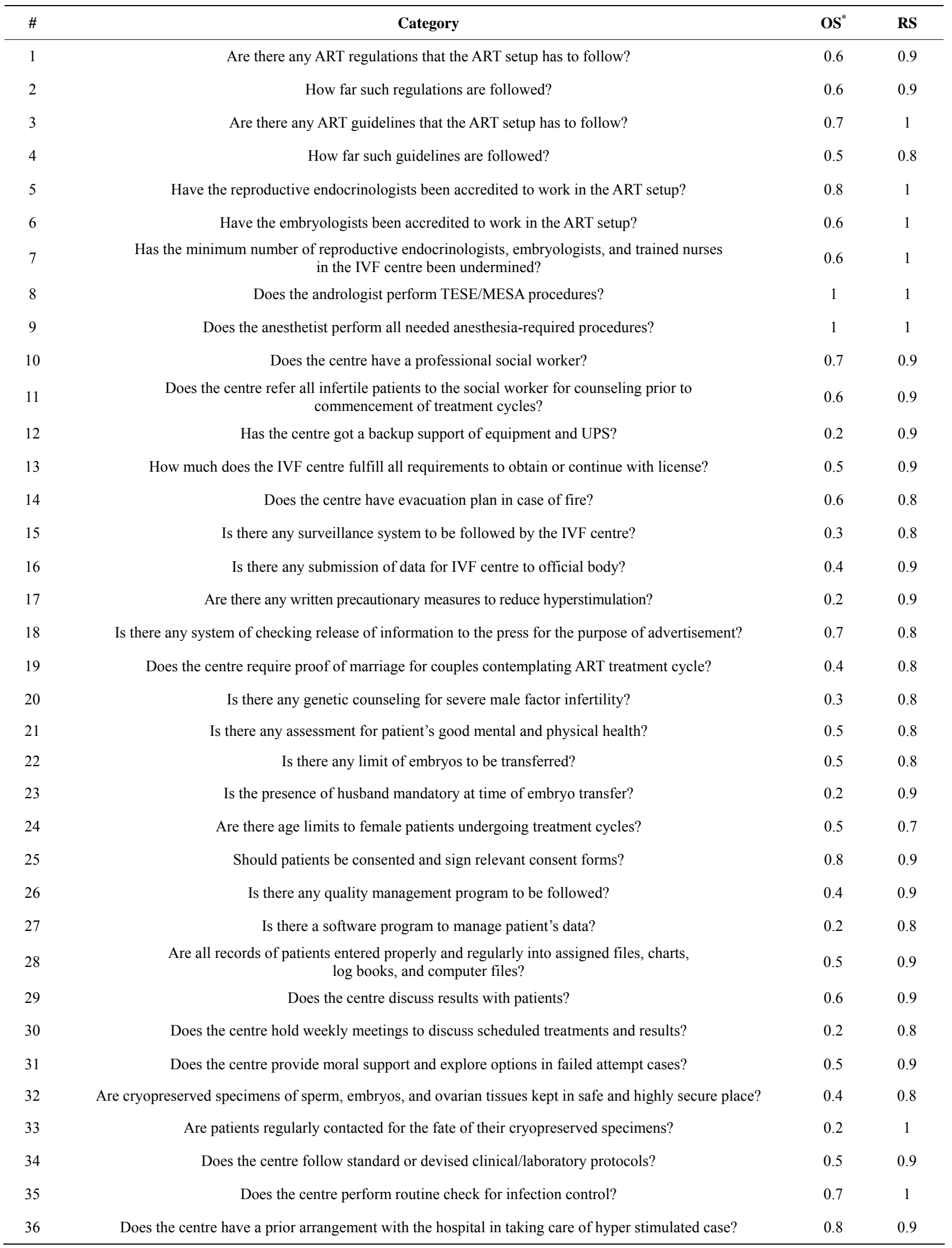




\section{Continued}

\begin{tabular}{|c|c|c|c|}
\hline 37 & Has the moral status of the conceptus been taken into consideration? & 0.4 & 1 \\
\hline 38 & Does the centre offer sex preselection based solely on clinical ground rather than patient's request? & 0.4 & 0.6 \\
\hline 39 & Are all handling, processing and utilization procedures of gametes are witnessed appropriately? & 0.4 & 0.9 \\
\hline 40 & Does the centre disapprove performing embryo reduction? & 0.6 & 1 \\
\hline 41 & Does the centre impose maximum limits for daily dose of FSH stimulation? & 0.6 & 0.8 \\
\hline 42 & Does the centre avoid performing ovarian stimulation with concomitant helpless TESE & 0.6 & 0.8 \\
\hline 43 & Is there any minimum resting period between successive IVF/ICSI treatment cycles? & 0.6 & 0.8 \\
\hline 44 & Is there any limit of offering IVF/ICSI treatment cycles per patient whatever the outcome? & 0.6 & 0.8 \\
\hline 45 & Is there any imposed limit towards higher cost of treatment cycle? & 0.6 & 1 \\
\hline & Total score & 23.6 & 39.6 \\
\hline & Mean & 0.52 & 0.88 \\
\hline & Standard deviation & 0.22 & 0.09 \\
\hline & P value (using paired t-test) & \multicolumn{2}{|c|}{0.001} \\
\hline
\end{tabular}

*OS, RS represent original and revamped settings respectively with highest score of 1.

Table 3. IVF/ICSI cycles and pregnancies by woman's age and number of embryo transferred for the period Sep 2004-Sep 2005.

\begin{tabular}{|c|c|c|c|c|c|c|c|c|c|}
\hline \multirow[b]{2}{*}{ Woman's Age } & \multicolumn{2}{|c|}{ IVF/ICSI cycles } & \multicolumn{2}{|l|}{ Transfers } & \multirow{2}{*}{$\begin{array}{c}\text { Pregnancies } \\
\text { Clinical }\end{array}$} & \multicolumn{4}{|c|}{ Deliveries (no babies) } \\
\hline & Initiated Total & Aspirations Total & Number of Embryos & Transfers & & Total & Singleton & Twin & Unknown \\
\hline \multirow[t]{5}{*}{$\leq 29$} & 71 & 67 & 1 & 6 & 1 & & 1 & & \\
\hline & & & 2 & 23 & 6 & & 3 & & \\
\hline & & & 3 & 26 & 6 & & 2 & 1 & 3 \\
\hline & & & 4 & 11 & 4 & & 1 & & \\
\hline & & & $\geq 5$ & 1 & & & & & \\
\hline NO ET & 4 & & Total & 67 & 17 & 8 & 7 & 1 & \\
\hline \multirow[t]{5}{*}{$30-34$} & 53 & 50 & 1 & 6 & & & & & \\
\hline & & & 2 & 6 & 3 & & 1 & & \\
\hline & & & 3 & 21 & 4 & & 1 & & 1 \\
\hline & & & 4 & 13 & 4 & & 2 & & \\
\hline & & & $\geq 5$ & 2 & & & & & \\
\hline NO ET & 3 & & Total & 48 & 13 & 4 & 4 & & \\
\hline \multirow[t]{5}{*}{$35-39$} & 49 & 44 & 1 & 10 & & & & & \\
\hline & & & 2 & 17 & 2 & & 1 & & 2 \\
\hline & & & 3 & 13 & 2 & & 1 & & \\
\hline & & & 4 & 3 & 1 & & 1 & & \\
\hline & & & $\geq 5$ & 1 & & & & & \\
\hline NO ET & 5 & & Total & 44 & 5 & 3 & 3 & & \\
\hline \multirow[t]{5}{*}{$\geq 40$} & 39 & 36 & 1 & 7 & 1 & & & & \\
\hline & & & 2 & 13 & 3 & & & 1 & \\
\hline & & & 3 & 11 & 1 & & 1 & & \\
\hline & & & 4 & 5 & & & & & \\
\hline & & & $\geq 5$ & & & & & & \\
\hline NO ET & 3 & & Total & 36 & 5 & 2 & 1 & 1 & \\
\hline TOTAL & 212 & 197 & & 195 & 40 & 17 & 15 & 2 & 6 \\
\hline
\end{tabular}


Table 4. IVF/ICSI cycles and pregnancies by woman's age and number of embryo transferred for the period Sep 2005-Sep 2006.

\begin{tabular}{|c|c|c|c|c|c|c|c|c|c|}
\hline \multirow{2}{*}{ Woman's Age } & \multicolumn{2}{|c|}{ IVF/ICSI cycles } & \multicolumn{2}{|c|}{ Transfers } & \multirow{2}{*}{$\begin{array}{c}\text { Pregnancies } \\
\text { Clinical }\end{array}$} & \multicolumn{4}{|c|}{ Deliveries (no babies) } \\
\hline & Initiated Total & Aspirations Total & Number of Embryos & Transfers & & Total & Singleton & Twin & Unknown \\
\hline \multirow[t]{5}{*}{$\leq 29$} & 68 & 64 & 1 & 6 & 2 & & 1 & & \\
\hline & & & 2 & 30 & 7 & & 4 & & 1 \\
\hline & & & 3 & 28 & 8 & & 5 & & 1 \\
\hline & & & 4 & 2 & 1 & & 1 & & \\
\hline & & & $\geq 5$ & & & & & & \\
\hline NO ET & 4 & & Total & 64 & 18 & 11 & 11 & & \\
\hline \multirow[t]{5}{*}{$30-34$} & 43 & 40 & 1 & 9 & 2 & & 1 & & \\
\hline & & & 2 & 19 & 5 & & 3 & & \\
\hline & & & 3 & 11 & 4 & & 2 & & \\
\hline & & & 4 & 1 & & & & & \\
\hline & & & $\geq 5$ & & & & & & \\
\hline NO ET & 3 & & Total & 40 & 11 & 6 & 6 & & \\
\hline \multirow[t]{5}{*}{$35-39$} & 57 & 55 & 1 & 10 & 2 & & 1 & & \\
\hline & & & 2 & 23 & 3 & & 2 & & 1 \\
\hline & & & 3 & 20 & 4 & & 3 & & 1 \\
\hline & & & 4 & 2 & 1 & & 1 & & \\
\hline & & & $\geq 5$ & & & & & & \\
\hline NO ET & 2 & & Total & 55 & 10 & 7 & 7 & & \\
\hline \multirow[t]{5}{*}{$\geq 40$} & 26 & 23 & 1 & 9 & 1 & & 1 & & \\
\hline & & & 2 & 10 & 3 & & 1 & & \\
\hline & & & 3 & 4 & 1 & & 1 & & \\
\hline & & & 4 & & & & & & \\
\hline & & & $\geq 5$ & & & & & & \\
\hline NO ET & 3 & & Total & 23 & 5 & 3 & 3 & & \\
\hline TOTAL & 194 & 182 & & 182 & $44^{*}$ & $27^{* *}$ & 27 & & 4 \\
\hline
\end{tabular}

${ }^{*} \mathrm{p}>0.05 ;{ }^{* *} \mathrm{p}<0.05$ (when statistically compared with those for the previous year as in Table 3).

$$
\mathrm{EVO}_{2}=0.91 \times 1.75 \times 0.88
$$

$$
\mathrm{EVO}_{2}=1.40
$$

The above calculations show a significant increase (40\%) in the EVO value of the centre, from 0.84 to 1.40 for the indicated periods. This is owed to the improvement in the success rates and the better compliance to the set standards although the economic performance has suffered a slight dip (by approximately $22 \%$ ), as a result of extra spending towards assets and lower utilization of treatment cycles. Moreover, there is a significant difference in the $C_{f}$ values before and after the amendments to the centre as indicated in Table 2. Although the pregnancy rates were not so significant, the birth rates were significant however, as shown in Tables 3 and $\mathbf{4}$.

\section{DISCUSSION}

Using the presented model not only the level of excellence in ART setup can be measured for the first time but the viability for its existence can also be evaluated. In this case, when the model was applied the results showed that there was approximately $40 \%$ gained improvement in EVO value due to certain changes which took place in the setup. Although there was a slight dip in economic 
performance as a result of reduced treatment cycles, the significant increase in compliance to standards conferred the rise in EVO value. However, there was no significant difference $(p>0.05)$ in the success rate variance between those two cases even though the live birth rate was significantly higher $(\mathrm{p}<0.05)$ in the revamped setting. Moreover, the EVO value could have been better if it were not for the newly opened ART centres that sliced off a share in the limited number of infertility cases in such small region. Nevertheless, EVO value adds up many advantages that can be used as an indicator of efficiency for utilization, self-assessment, help in improving the cost-effectiveness of the treatment services offered by the setup, help in market research, planning reinvestment/new investment, providing the national ART Registry from available data, and measuring the safety and efficacy of the treating centre.

\section{CONCLUSIONS}

The presence of many ART centres/clinics in a particular region has the advantage of giving its patients more choices for the treatment of infertility and therefore more utilization of such services. However, too many centres per region may have detrimental effects; causing lower returns which will not help research and development programs especially when the cost of treatment is rather low (see Table 1 for comparative study). As can also be depicted from the table, certain countries cannot afford clinical and scientific research and development programs since the return of the investment is barely enough to get the setup going with a small margin of profit. Despite the higher infant mortality rates of the Middle East countries, the level of utilization of IVF cycles is not significantly different from those of the developed countries; contrary to previous study [8].

When considering the investment at a larger scale as evident from the larger and more prosperous population with excellent revenue of relatively high number of treatment cycles (Table 1), it looks more feasible to conduct research work in the ART field. Also, additional spending on development programs will unequivocally allow the concerned ART centre to achieve excellent quality of treatment, high standard of care, and the resulting success rates, rather than cutting corners for the benefit of economic survival of the centre. Hence, applying the presented mathematical model provides many answers to such lengthy argument.

\section{ACKNOWLEDGEMENTS}

I would like to express my sincere thanks to Dr. Ammar, FAMCO, University of Dammam and Dr Amani Abrahim, College of Applied Medical Sciences, University of Dammam for their assistance in the bio-statistical analysis.

\section{REFERENCES}

[1] Rhoton-Vlasak, A. (2000) Infections and infertility. Primary Care Update Ob/GYNS, 1, 200-206.

[2] Palagiano, A., et al. (2004) FSH: Urinary and recombinant. European Journal of Obstetrics, Gynecology and Reproductive Biology, 1, S30-S33. doi:10.1016/j.ejogrb.2004.01.023

[3] Devlin, N. and Parkin, D. (2003) Funding fertility: Issues in the allocation and distribution of resources to assisted reproduction technologies. Human Fertility (Cambridge), 6, 2-6. doi:10.1080/1464770312331369153

[4] Van Voorhis, B.J. (2003) Evaluating economic studies in reproductive medicine. Seminars in Reproductive Medicine, 21, 85-93. doi:10.1055/s-2003-39998

[5] Eskandarani, H.A. (1996) Assisted reproduction technology: State-of the-art. The Islamic Educational, Scientific and Cultural Organization (ISESCO) Publication, Rabat, 73-75.

[6] Eskandarani, H.A. (2007) A code of practice is overdue for assisted reproductive technology in the GCC countries: Some comments and suggestions. Qatar Medical Journal, 16, 16-19.

[7] Eskandarani, H.A. (2010) A bahraini registry of assisted reproductive technology for period of 2000-2006: Hoping to stave off reporting stalemate. Journal of Reproduction and Contraception, 21, 101-110. doi:10.1016/S1001-7844(10)60018-1

[8] Collins, J.A. (2002) An international survey of the health economics of IVF and ICSI. Human Reproduction Update, 8, 265-277. doi:10.1093/humupd/8.3.265 